\title{
Epitope Analysis of Cerebrospinal Fluid IgG in Japanese Multiple Sclerosis Patients Using Phage Display Method
}

\author{
Juichi Fujimori, ${ }^{1}$ Ichiro Nakashima, ${ }^{1}$ Kazuo Fujihara, ${ }^{1}$ Tatsuro Misu, ${ }^{1}$ Shigeru Sato, ${ }^{2}$ \\ and Yasuto Itoyama ${ }^{1}$ \\ ${ }^{1}$ Department of Neurology, Tohoku University School of Medicine, 1-1 Seiryo-machi, Aoba-ku, Sendai 980-8574, Japan \\ ${ }^{2}$ Department of Neurology, Kohnan Hospital, Sendai 982-8523, Japan \\ Correspondence should be addressed to Juichi Fujimori, j-fujimori@tohoku-knhp.ne.jp
}

Received 16 August 2011; Revised 21 September 2011; Accepted 21 September 2011

Academic Editor: H. P. Hartung

Copyright (๑) 2011 Juichi Fujimori et al. This is an open access article distributed under the Creative Commons Attribution License, which permits unrestricted use, distribution, and reproduction in any medium, provided the original work is properly cited.

To investigate the antigen recognized by cerebrospinal fluid (CSF) high affinity IgG in patients with multiple sclerosis (MS), the phage display method was applied to the CSF from 15 MS and 10 control patients. Peptide sequences recognized by MS and control CSF IgG were individual specific, and no common motif was found. Peptide sequences frequently showed homology to various kinds of amino acid sequences of ubiquitous viruses such as epstein barr virus (EBV) and herpes simplex virus (HSV), although the frequency was not specific to MS patients. MS CSF IgG may recognize various types of ubiquitous viral antigen and may be increased by a bystander response.

\section{Introduction}

In multiple sclerosis (MS), the pathogenetic events that result in immune cell infiltration, multifocal demyelination, and axonal loss are still under debate. Recognition has emerged that both mutually interacting cellular and humoral immune components may contribute to immune-mediated demyelination in human disorders [1].

Normal cerebrospinal fluid (CSF) has an IgG content that is less than $13 \%$ of the total protein. In MS, there is an unexplained elevation of IgG in the CSF from 15\% to $30 \%$, visualized as oligoclonal bands $(\mathrm{OB})$ after electrophoresis [2].

Although the humoral immune response is implicated in MS, its role in the pathogenesis has not been determined precisely. Most researchers seem to consider MS CSF IgG or oligoclonal IgG band to be a nonspecific bystander response. On the other hand, several laboratories studied the antigen-binding regions of antibodies found in MS brain demyelinative plaques and cerebrospinal fluid and revealed (a) limited germline expression, results not expected for a random bystander response; (b) features consistent with a specific antigen-targeted process; (c) the clonal expansion of populations of B lymphocytes in MS [2]. Furthermore, the target antigen of MS CSF IgG has not been determined.

We have successfully confirmed that CSF IgG from HTLV-I-associated myelopathy/tropical spastic paraparesis (HAM/TSP) patients selected motifs that were highly homologous to the causative microorganism "HTLV-I" from a 12mer random peptide library (RPL) using the phage display method [3]. We tried to find the target antigen of CSF IgG in MS patients using the same method as we did in the cases of HAM/TSP.

\section{Materials and Methods}

2.1. Patients and Controls. CSF samples from $15 \mathrm{MS}$ patients (13 women and 2 men, mean age 37 (range, 21-60) years,) were analyzed. The mean onset age of the patients was 29 years, and the mean duration of the disease was 8 years. The mean IgG index was 1.07. All MS patients fulfilled McDonald's criteria and the samples were obtained during acute relapses of the disease. By testing with isoelectric focusing and IgG immunofixation method, 14 patients were 
found to be $\mathrm{OB}$ positive and one patient (MS7) was OB negative. In a single patient (MS6), CSF samples collected at three different points of time were studied.

The control group was comprised of 5 patients with psychosomatoform disorder and 5 patients with headache. CSF samples from 10 control patients ( 5 men and 5 women), mean age 28.7 years (range, 14-48), were analyzed.

2.2. Phage Display Method. One hundred microliters of Dynabeads Protein A (Dynal Biotech ASA, Oslo, Norway) were incubated with $300 \mu \mathrm{L}$ of CSF overnight at $4^{\circ} \mathrm{C}$. The IgG-bead complexes were incubated with $10 \mu \mathrm{L}$ of a 12 mer random peptide library containing approximately $4 \times$ $10^{10}$ particles (Ph.D-12, Phage Display Peptide Library Kit, New England Biolabs, Beverly, MA) for 60 minutes at room temperature. After washing twice with phosphate-buffered saline containing $0.1 \%$ Tween-20, bound phages were eluted and amplified by infecting Escherichia coli (ER2738 strain) and incubated for $4.5 \mathrm{~h}$ at $37^{\circ} \mathrm{C}$. Bacterial cells were removed by centrifugation, and the amplified phages were purified by polyethylene glycol (PEG) precipitation. The phage solution was titrated to determine the concentration. Newly made IgG-bead complexes were mixed for 60 minutes with the $2 \times 10^{11}$ phage particles derived from the first round phage selection. After washing six times, bound phages were eluted. Thirty-three clones from the second round of phage selection were sequenced for DNA to determine the displayed 12mer amino acids. All these procedures were based on the manufacturer's protocol. The selected phage clones were numbered in each patient. For example, p7_33 denoted the 33rd clone in patient MS7.

2.3. Homology Search for Selected Amino Acid Sequence Motif. We searched for a common peptide motif, a specific sequence pattern that occurs repeatedly in a group of peptide sequences, using Multiple Expectation Maximum for Motif Elicitation (MEME) version 3.0 (http://meme.sdsc.edu/ meme/cgi-bin/meme.cgi/) [4]. BLAST (Search for short nearly exact matches, National Center for Biotechnology Information) was searched for proteins containing homologous sequences.

\section{Results}

3.1. Phage Display. CSF from 13 out of 15 MS patients and CSF from 8 out of 10 control patients selected one or more common peptide motifs, but the peptide motifs were basically different from one patient to another (Tables 1 and 2 ). We tried to detect common peptide motifs in a total of 495 motifs derived from 15 MS patients, but we could not detect any MS-specific common peptide motifs.

3.2. Homology Search. In the BLAST search, we could not detect microbial agents or human molecules that significantly correlated with MS patients. However, we focused on non-human sequences with the intention of obtaining insight into etiological microbial agents of MS. The detected peptide motifs were frequently homologous to the components of herpes simplex virus 1 (HSV-1), cytomegalovirus
(CMV), and EBV, while these motifs were rarely homologous to the components of measles virus, rubella virus, and varicella zoster virus. As for putative myelin autoantigens in MS, the motif of myelin basic protein was detected in only one patient (MS5). In patient MS6, identical peptide motifs homologous to the components of EBV were repeatedly detected from the 3 CSF samples (Table 3 ). The Expect value $(E)$ is a parameter that describes the number of hits one can "expect" to see by chance when searching a database of a particular size. The lower the $E$-value, or the closer it is to zero, the more "significant" the match is. The E-values of the three peptide motifs SPxxMH, DPYQxP, and PYxxYQxP for EBV were 0.32, 0.008, 0.028, respectively, when we used BLAST limiting the organism to viruses.

\section{Discussion}

Using molecular immunologic strategies, there have been several analyses of intrathecally synthesized oligoclonally expanded immunogloblins with high affinity to identify specific antigens. The screening of libraries expressing protein products derived from chronic MS plaque messenger RNA with antibodies purified from plaques, CSF, or serum of patients with MS has not thus far revealed the antigenic targets of the MS antibody response [2].

The phage display method utilizes random peptide libraries displayed on phages expressing a large collection of peptide sequences $\left(10^{8}\right.$ or more) that mimic linear or conformational epitopes of folded protein domains, and even carbohydrate structures [5-7]. We analyzed the binding motifs of IgG in the CSF of HAM/TSP patients using the phage library method [3]. As a result, sequences highly homologous to HTLV-I gp46 were the common linear epitopes of high affinity-IgG exclusively detected in both CSF and sera of the patients.

A few laboratories have previously reported the selection from RPL of several peptides that bind to antibodies present in the CSF of Caucasian MS patients. However, the results are still inconsistent. Rand and colleagues reported that CSF IgG antibody selected the amino acid sequence motif RRPFF from a random peptide library in five out of 14 American patients with MS. The RRPFF motif is found in EBV nuclear antigen [8]. Cortese and colleagues carried out a similar study of CSF from Italian patients with MS using the phage display method. Three different candidate amino acid motifs selected by CSF from two patients with MS were tested and, whereas they were recognized with equal frequency by serum from both MS and control patients, CSF from only 4 out of 55 patients with MS reacted with one of these three motifs. With another experiment, they concluded that CSF-enriched antibodies did not share specificities among MS patients $[9,10]$. We tried to find a common motif with a 12 -mer peptide library in a larger cohort than those in the previous studies.

After confirming that the phage display method combined with MEME and BLAST search could detect the target antigen of CSF IgG from HAM/TSP patients, we investigated the potential target antigens recognized by high affinity IgG in MS CSF using the phage display method. However, 


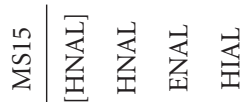

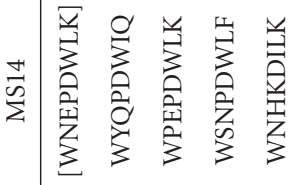

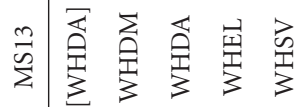

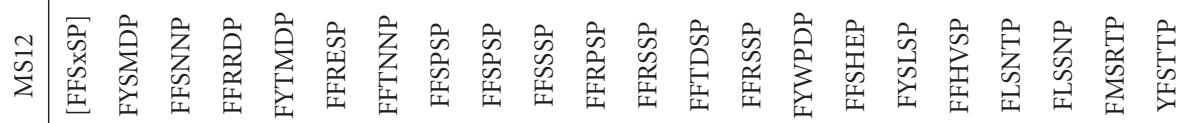

$\overline{5}$
$\sum$
$\stackrel{5}{\infty}$
$\stackrel{5}{\infty}$

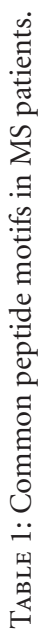

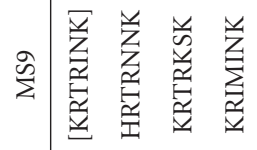

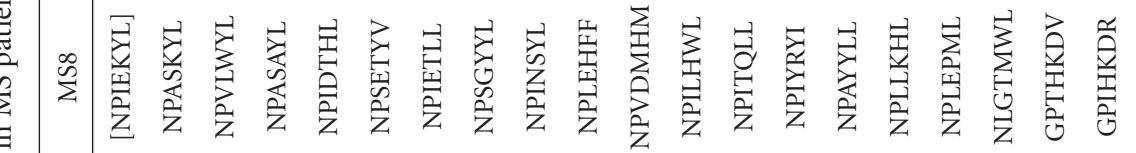



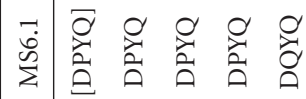

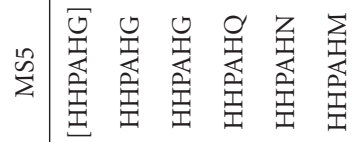



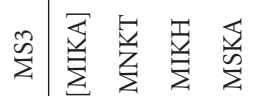

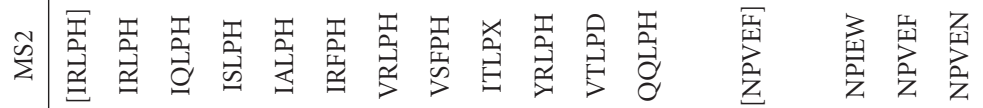

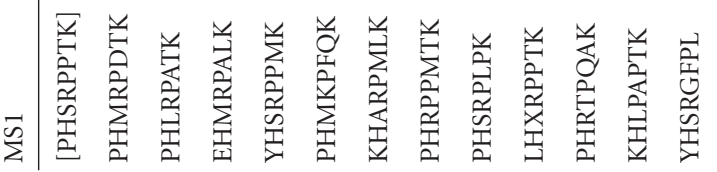

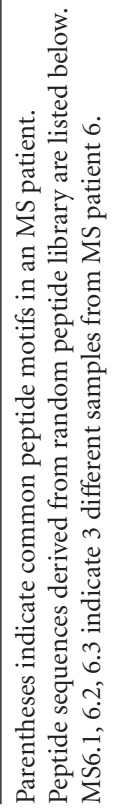


TABLE 2: Common peptide motifs in control group.

\begin{tabular}{|c|c|c|c|c|c|c|c|c|c|}
\hline Cont 1 & Cont 2 & Cont 3 & Cont 4 & Cont 5 & Cont 6 & Cont 7 & Cont 8 & Cont 9 & Cont 10 \\
\hline & [IHHPAHG] & [NPIEAF] & [KPPNP] & [GVTDY] & & [HILHNPF] & [GPALSxSE] & [KPPNPS] & [IYPTTLPW] \\
\hline & IHHPAHG & NPVENF & KPPNP & GVQDY & & HILHNPF & RPALSMYE & KPPNPM & IYRTTLPW \\
\hline & MHHPAHQ & NHCCAF & KPPNP & GVHDY & & HSLHNPF & RPALSMYE & KPPNPM & IYPTTLPW \\
\hline & SHHPAHG & NPIELW & KPPNP & GMTDY & & HLLHSPF & MPALSVSE & KPPNPS & IYTVTLPW \\
\hline & NHHPAHM & NPIVNY & KPPNP & GLIDY & & HYAHNPF & GPALSHSE & KPPNPS & \\
\hline & & NPVLAW & KPPNP & QITDY & & HNHHNPF & RPALTPQE & KPPNPS & [YLDL] \\
\hline & [YLPK] & NPIEKL & KPPNP & & & HIMHDPL & QPALSSTE & KPPNPE & \\
\hline & & NPVLTF & & [LPHWSP] & & HNLHSPW & MPMLSAEE & KPPNPT & YYDL \\
\hline & YLPK & & [SLQSLRAAAM] & & & & SPHISNKE & KPPNPL & YLDL \\
\hline & YEPQ & & & LPHWSP & & & GPSLLNVE & & YPDL \\
\hline & YLSK & & SIQSLRTAFM & LPHWSP & & & TPALHRLE & & \\
\hline & YLPT & & SLQSLRGASA & LPHWMP & & & VPTLNVAE & & \\
\hline & & & SLSSLRAAAF & LPHWQP & & & GPSLHPSE & & \\
\hline & & & SVRSLHERVM & LPHWSF & & & MPGLPSQE & & \\
\hline & & & & IRHWVP & & & SPATYFLE & & \\
\hline & & & & & & & SPQLSAHG & & \\
\hline & & & & & & & [PWSK] & & \\
\hline & & & & & & & PWSK & & \\
\hline & & & & & & & PWSK & & \\
\hline & & & & & & & PWSK & & \\
\hline & & & & & & & PWSK & & \\
\hline & & & & & & & PWSK & & \\
\hline & & & & & & & PWSK & & \\
\hline & & & & & & & PWSK & & \\
\hline & & & & & & & PWSK & & \\
\hline
\end{tabular}

Parentheses indicate common peptide motifs in a control case.

Peptide sequences derived from random peptide library are listed below.

TABle 3: Common peptide motifs derived from 3 CSF samples of MS6.

\begin{tabular}{|c|c|c|c|}
\hline & \multicolumn{3}{|c|}{ Peptide motifs } \\
\hline & $(\mathrm{SPxxMH})$ & (DPYQxP) & (PYxxYQxP) \\
\hline \multirow{5}{*}{$\begin{array}{l}1999.12 \\
\text { (Remission) }\end{array}$} & SPPKMH & DPYQVP & PYEFYQYP \\
\hline & SPKSMH & GPYQDP & PYLNYQHP \\
\hline & SPPLMH & DPYQLP & \\
\hline & SPRNMH & & \\
\hline & SPVHMH & & \\
\hline \multirow{4}{*}{$\begin{array}{l}2000.1 \\
\text { (Relapse) }\end{array}$} & SPRIDH & DPYQLP & PYTGYQ \\
\hline & SPPLMH & DPYQSL & PYAGYQ \\
\hline & & DPYQVY & \\
\hline & & DQYQQP & \\
\hline \multirow{4}{*}{$\begin{array}{l}2000.10 \\
\text { (Remission) }\end{array}$} & SPYHSH & DYYQQH & PYSPYQLP \\
\hline & SPRNMH & DPYQTA & PYNLYQTP \\
\hline & SPGNMH & DPYQLP & TYGAYQLA \\
\hline & & & PYAHYQPY \\
\hline EBV component & $\begin{array}{c}\text { IYYSPSIMHR } \\
\text { (EBV VP19) }\end{array}$ & $\begin{array}{l}\text { PDPYQLPFA } \\
\text { (EBV ZEBRA) }\end{array}$ & $\begin{array}{l}\text { APYQGYQEPP } \\
\text { (EBV EBNA 6) }\end{array}$ \\
\hline
\end{tabular}

Bold-faced letter indicates common peptide motifs.

we could not detect a peptide sequence or antigen that is recognized exclusively by CSF IgG from most of MS patients.
One possible reason that we could not detect an epitope is that the MS CSF IgG may recognize a conformational epitope or nonprotein antigens. Another possible reason is that MS CSF IgG may recognize epitopes with lower affinity that may not exist dominantly compared to HAM CSF IgG. In our study, CSF IgG from two MS patients with OCB could not recognize common peptide motifs. When exploring MS CSF IgG epitopes using the phage display method, it might be necessary to sequence much larger numbers of phage clones and to increase the number of pannings. It is still difficult to conclude that the MS CSF IgG antigen does not exist. However, considering the fact that no MS antigen has been determined so far, MS CSF IgG may not recognize a single MS antigen, differing from HAM/TSP CSF IgG which recognizes HTLV-I. Also, the fact that the common peptide motifs detected in MS and control groups were not significantly different may suggest that MS CSF IgG may recognize ubiquitous antigens, also differing from the case of HAM/TSP CSF IgG.

Interestingly, in patient MS6, identical peptide motifs homologous to the components of EBV were repeatedly detected from the 3 CSF samples. The $E$-values of the three peptide motifs from MS-6 for EBV were 0.008-0.32. In the former study, the $E$-values of the common peptide motifs from HAM/TSP CSF IgG for HTLV-I were 0.15-0.95. The peptide motifs were indicative of the EBV specificity. MS 
CSF IgG may recognize antigens persistently and epitope spreading may not occur in some patients.

Recently, Meinl and colleagues reported that inflammation in the central nervous system (CNS) provides a unique, B-cell-friendly environment, in which B lineage cells, notably long-lived plasma cells, can survive for many years. These long-lived plasma cells may produce oligoclonal IgG bands. CNS resident cells (mainly astrocytes and microglia) produce mediators such as chemokines, cytokines, and adhesion molecules that promote the local survival of plasma cells [11]. Whether the antibodies derived from these longlived plasma cells target specific antigens or nonspecific ubiquitous antigens remains to be determined.

MS CSF IgG is considered to be oligoclonally increased, and the factor that evokes this response may not be a single, rare MS antigen. Other factors that survive specific B cells may evoke this response. Such factors, rather than a single rare antigen, may be common in the pathogenesis of MS. Further analysis of such factors may be critical to understanding the pathogenesis of MS.

\section{Acknowledgments}

The authors thank Dr. Masahiro Yamamoto and Dr. Naoki Yamamoto from Tokyo Medical and Dental University for their technical assistance. The authors thank Mr. Brent Bell for reading the paper. This work was supported by Grants-inAid for General Scientific Research (13470131 and 13670626) from the Ministry of Education, Science, Culture, Science and Technology, a grant from the Neuroimmunological Research Committee.

\section{References}

[1] J. J. Archelos, M. K. Storch, and H. P. Hartung, "The role of B cells and autoantibodies in multiple sclerosis," Annals of Neurology, vol. 47, no. 6, pp. 694-706, 2000.

[2] D. H. Gilden, M. P. Burgoon, B. K. Kleinschmidt-DeMasters et al., "Molecular immunologic strategies to identify antigens and B-cell responses unique to multiple sclerosis," Archives of Neurology, vol. 58, no. 1, pp. 43-48, 2001.

[3] J. Fujimori, I. Nakashima, K. Fujihara et al., "Epitope analysis of the cerebrospinal fluid IgG in HTLV-I associated myelopathy patients using phage display method," Journal of Neuroimmunology, vol. 152, no. 1-2, pp. 140-146, 2004.

[4] T. L. Bailey and C. Elkan, "Fitting a mixture model by expectation maximization to discover motifs in biopolymers," in Proceedings of the 2nd International Conference on Intelligent Systems for Molecular Biology, pp. 28-36, AAAI Press, Menlo Park, Calif, USA, 1994.

[5] X. Chen, G. Scala, I. Quinto et al., "Protection of rhesus macaques against disease progression from pathogenic SHIV89.6PD by vaccination with phage-displayed HIV-1 epitopes," Nature Medicine, vol. 7, no. 11, pp. 1225-1231, 2001.

[6] G. P. Smith and V. A. Petrenko, "Phage display," Chemical Reviews, vol. 97, no. 2, pp. 391-410, 1997.

[7] G. P. Smith and J. K. Scott, "Libraries of peptides and proteins displayed on filamentous phage," Methods in Enzymology, vol. 217, pp. 228-257, 1993.

[8] K. H. Rand, H. Houck, N. D. Denslow, and K. M. Heilman, "Molecular approach to find target(s) for oligoclonal bands in multiple sclerosis," Journal of Neurology Neurosurgery and Psychiatry, vol. 65, no. 1, pp. 48-55, 1998.

[9] I. Cortese, S. Capone, S. Luchetti, L. M. E. Grimaldi, A. Nicosia, and R. Cortese, "CSF-enriched antibodies do not share specificities among MS patients," Multiple Sclerosis, vol. 4, no. 3, pp. 118-123, 1998.

[10] I. Cortese, R. Tafi, L. M. E. Grimaldi, G. Martino, A. Nicosia, and R. Cortese, "Identification of peptides specific for cerebrospinal fluid antibodies in multiple sclerosis by using phage libraries," Proceedings of the National Academy of Sciences of the United States of America, vol. 93, no. 20, pp. 11063-11067, 1996.

[11] E. Meinl, M. Krumbholz, and R. Hohlfeld, "B lineage cells in the inflammatory central nervous system environment: migration, maintenance, local antibody production, and therapeutic modulation," Annals of Neurology, vol. 59, no. 6, pp. 880-892, 2006. 




The Scientific World Journal
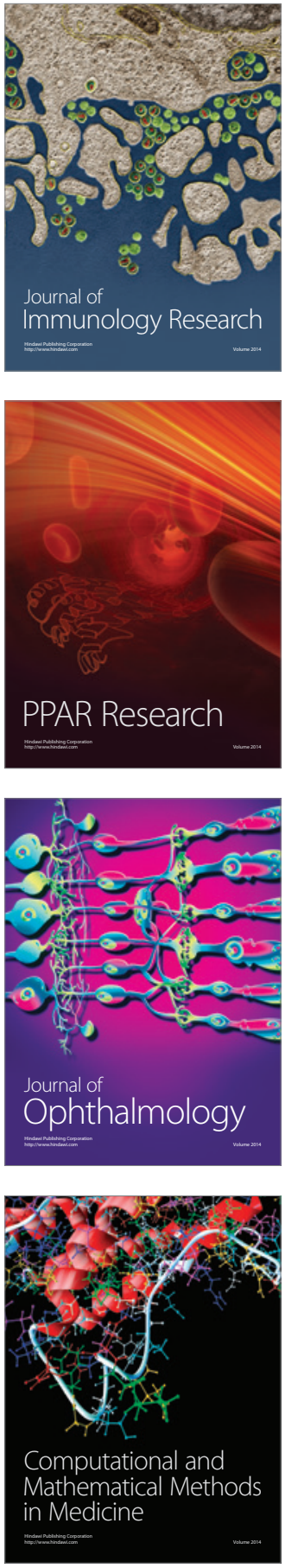

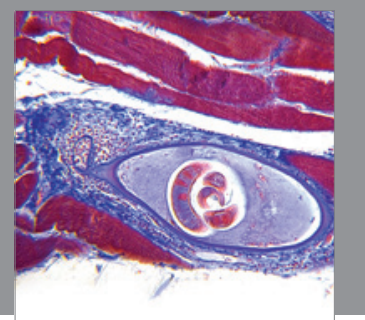

Gastroenterology

Research and Practice
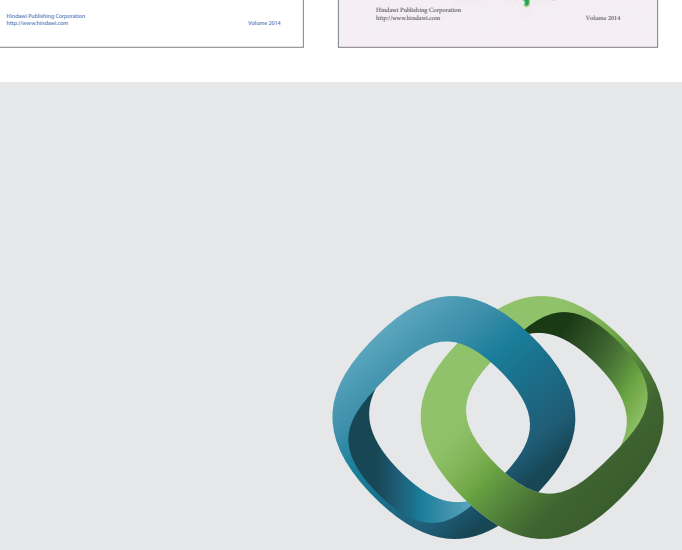

\section{Hindawi}

Submit your manuscripts at

http://www.hindawi.com
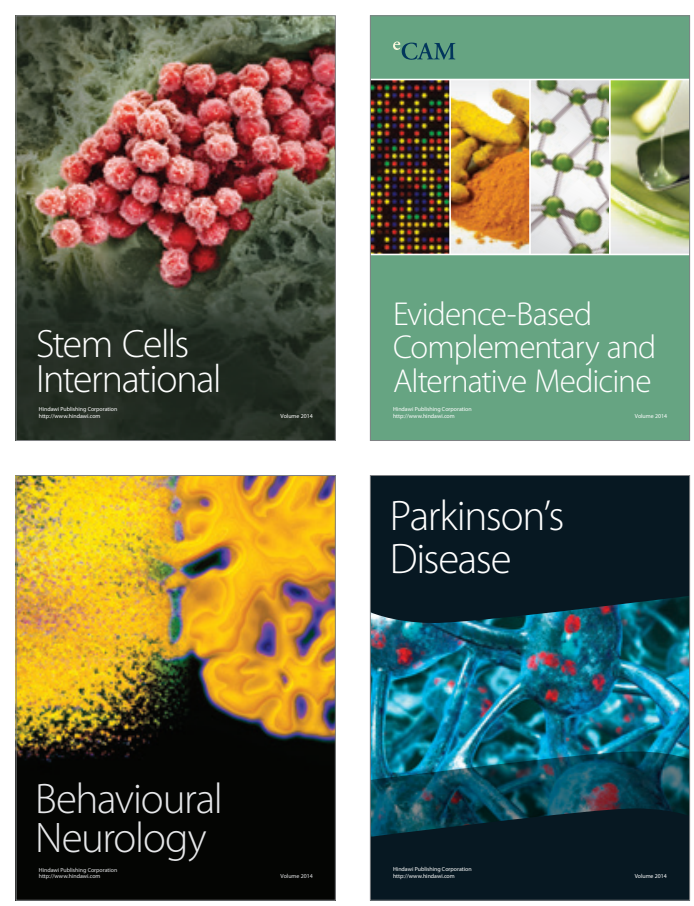

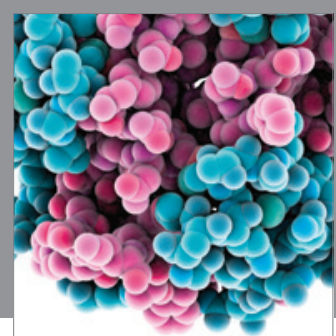

Journal of
Diabetes Research

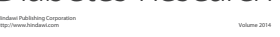

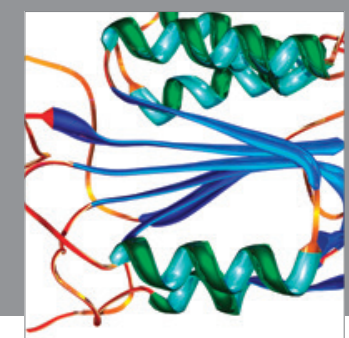

Disease Markers
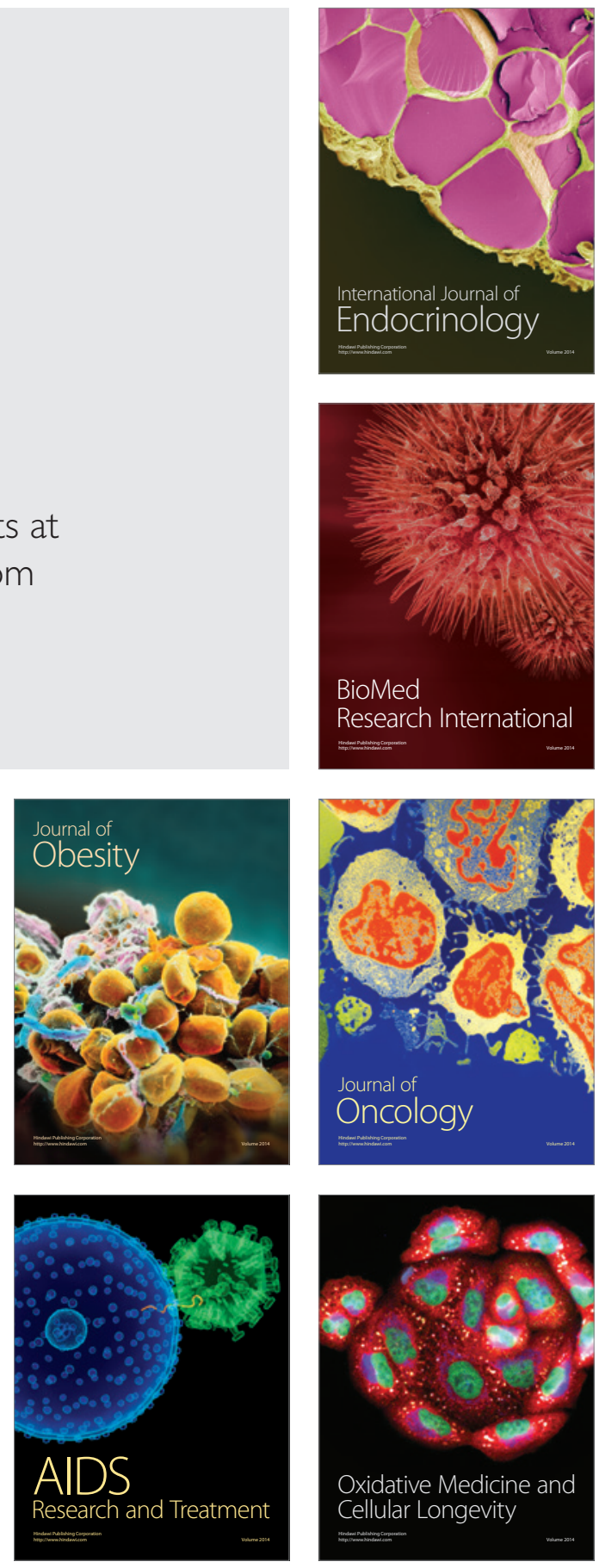